\title{
High-pressure and high-temperature differential scanning calorimeter for combined pressure-concentration-temperature measurements of hydrides
}

\author{
Ph. Mauron, ${ }^{1}$ M. Bielmann, ${ }^{1}$ V. Bissig, ${ }^{2}$ A. Remhof,${ }^{1}$ and A. Züttel ${ }^{1}$ \\ ${ }^{1}$ Empa. Swiss Federal Laboratories for Materials Testing and Research, Laboratory of Hydrogen and Energy, \\ Überlandstrasse 129, CH-8600 Dübendorf, Switzerland \\ ${ }^{2}$ Empa. Swiss Federal Laboratories for Materials Testing and Research, Laboratory of Joining and Interface \\ Technology, Überlandstrasse 129, CH-8600 Dübendorf, Switzerland
}

(Received 19 May 2009; accepted 30 August 2009; published online 24 September 2009)

\begin{abstract}
The design and construction of a high-pressure $(200 \mathrm{bar})$ and high-temperature $\left(600{ }^{\circ} \mathrm{C}\right)$ heat-flow differential scanning calorimeter (DSC) for the in situ investigation of the hydrogenation and dehydrogenation reactions of hydrides is presented. In combination with a pressure-concentration-temperature $(\mathrm{pcT})$ system, simultaneous thermodynamic and volumetric measurements become accessible. Due to the high thermal conductivity of hydrogen, only the sample cell and the reference cell are exposed to hydrogen and the remaining system is under ambient conditions. This separation has the advantage that the calibration factor is independent of the hydrogen pressure. The internal empty volume of the combined system is as low as possible to maximize the precision of the pcT measurements. The calorimetric block of the DSC is designed with a silver/copper alloy and the temperature measurements are made resistively with platinum temperature sensors (Pt 100). The instrument was calibrated and its operability was successfully studied on the example of the hydrogen sorption behavior of $\mathrm{LaNi}_{5}$. () 2009 American Institute of Physics. [doi:10.1063/1.3233939]
\end{abstract}

\section{INTRODUCTION}

In view of an application of hydrides as hydrogen storage medium in stationary and mobile applications, the knowledge and design of the thermodynamic properties are crucial in order to have a tank that can be operated at moderate pressure and temperature conditions. ${ }^{1}$ A high-pressure differential scanning calorimeter (DSC) in combination with a pressure-concentration-temperature (pcT) system is an ideal tool to investigate the de- and rehydrogenation enthalpies of hydrides due to the fact that the hydrogen concentration can be determined at the same time. The pcT system can be used as dosing system to control the pressure during experiments, e.g., for isobaric measurements. Furthermore structural phase transformations and hydrogen sorption events can be separated and identified.

\section{A. Metal hydrides and thermodynamics}

Metal hydrides are metals or alloys that have the property to dissolve and absorb a large amount of hydrogen. A well-known example is the alloy $\mathrm{LaNi}_{5}$ that can absorb six hydrogen atoms per formula unit to form $\mathrm{LaNi}_{5} \mathrm{H}_{6}{ }^{2}$ This reaction is reversible at moderate pressure and temperature conditions: $\mathrm{LaNi}_{5}+3 \mathrm{H}_{2} \leftrightarrow \mathrm{LaNi}_{5} \mathrm{H}_{6}$. During hydrogenation of a metal at a constant temperature and at low hydrogen concentrations first the so called $\alpha$-phase is formed that is a solid solution of hydrogen in the metal. At higher concentrations the metal hydride phase ( $\beta$-phase) is formed. During the formation of the $\beta$-phase the pressure is constant and forms a plateau (cf. Fig. 7). By measuring isotherms at dif- ferent temperatures and by plotting the equilibrium pressure $p_{\text {eq }}$ as a function of the inverse temperature $1 / T$ in a so called Van 't Hoff plot, the thermodynamic parameters as the enthalpy $\Delta H$ and entropy $\Delta S$ of the de- and rehydrogenation reaction from the $\alpha$-phase to the $\beta$-phase can be determined with the Van 't Hoff equation

$$
\ln \left(\frac{p_{\text {eq }}}{p_{0}}\right)=\frac{\Delta H}{R} \cdot \frac{1}{T}-\frac{\Delta S}{R}
$$

with $p_{0}=1.01325 \mathrm{bar}$ and $R=8.31447 \mathrm{~J} /(\mathrm{mol} \mathrm{K})$ the ideal gas constant. For $\mathrm{LaNi}_{5} \Delta H=-30.8 \mathrm{~kJ} / \mathrm{mol} \mathrm{H}$ and $\Delta S=$ $-108 \mathrm{~J} /\left(\mathrm{K} \mathrm{mol} \mathrm{H}_{2}\right)$ (Ref. 3) leading to an equilibrium temperature of $T_{\mathrm{eq}}=\Delta H / \Delta S=285 \mathrm{~K}$ corresponding to $12{ }^{\circ} \mathrm{C}$ at $p_{\text {eq }}=p_{0}$.

\section{B. pcT instrument and measuring method}

The pcT system used in our experiments uses a mass flow controller and the pcT isotherms are measured in a dynamic mode. During a hydrogenation experiment a constant hydrogen flow is applied to the sample and the increasing pressure is recorded as a function of the hydrogen concentration that can be calculated from the flow. The measured isotherms have to be corrected by the system volume that should be as small as possible. This type of pcT system and the measuring method are described in detail by Bielmann $e t$ $a l^{4}$ For this dynamic measurement method, several isotherms at different flow rates have to be measured and the equilibrium pressure $p_{\text {eq }}$ can be determined by extrapolating $\ln \left(p_{\mathrm{pl}} / p_{0}\right)$ of the plateau pressures $p_{\mathrm{pl}}$ to zero flow. 


\section{DSC method}

\section{Isobaric measurements}

A DSC allows the direct or indirect determination of the enthalpy of the de- and rehydrogenation reaction. In the indirect method, the hydride is heated or cooled at a constant heating rate and at a constant hydrogen pressure. If the reaction rate is very fast, compared to the heating rate, the peak temperature, ${ }^{5}$ or onset temperature ${ }^{6}$ can be taken as the equilibrium temperature for the given hydrogen pressure. The equilibrium temperature as a function of the applied pressure is then represented in a Van 't Hoff plot from which the thermodynamic parameters can be determined. In the direct method the integral of the DSC peak can be taken as shown by Borgschulte $e t a l^{7}$ for the palladium-hydrogen system.

\section{Isothermal measurements}

In general, the heat in differential form $d Q_{m}$ measured in a calorimeter can be written as ${ }^{8}$

$$
\begin{aligned}
d Q_{m}= & {\left[\left(\frac{\partial H}{\partial p}\right)-V\right]_{T, \xi} d p+\left(\frac{\partial H}{\partial T}\right)_{p, \xi} d T+\left(\frac{\partial H}{\partial \xi}\right)_{T, p} d \xi } \\
& -\sum_{i} d E_{i},
\end{aligned}
$$

where the enthalpy $H$ is a function of the pressure $p$, temperature $T$, and composition $\xi$.

When measuring a pcT curve of a metal hydride as, e.g., $\mathrm{LaNi}_{5}$, the sample is at a constant temperature and the above expression can be simplified as discussed as follows. In the case of a metal hydride the investigated system consists of a solid and a gas phase and consequently $H$ is the sum of the enthalpy of both phases. The first term in Eq. (2) can be considered zero because for metal hydrides the pressure is almost constant over a wide range of hydrogen concentration $(\sim 90 \%)$ in the two phase region. Considerable changes only occur at low hydrogen concentrations in the pure $\alpha$-phase and at high concentrations in the pure $\beta$-phase. Furthermore, in our case the sample and reference volume are small $\left(1.4 \mathrm{~cm}^{3}\right)$ and if the gas volume in the sample and reference holder are equal, the effect of $V \cdot d p$ of the gas phase is subtracted out in the DSC signal. ${ }^{11}$ The effect of $d p$ on the solid phase can be neglected. The same argument holds for the Joule-Thomson effect of the hydrogen gas which coefficient is proportional to $(\partial H / \partial p)_{T, \xi}$. For isothermal $(d T=0)$ measurements, the second term, representing the heat capacity is also zero. In the last term, $d E_{i}$ represents other energy forms as, e.g., surface heat etc. that are not present in the investigated system or can be neglected. Equation (2) therefore simplifies to $d Q_{m}=(\partial H / \partial \xi)_{T, p} d \xi$ and consequently the integrated value corresponds to the enthalpy of reaction of the hydride formation.

With a DSC, the reaction enthalpy of one isotherm and the evolution of the enthalpy during the reaction can be determined. ${ }^{9-11}$ In the case of metal hydrides the integral over the whole reaction gives the total enthalpy change and not only the enthalpy from the $\alpha$-phase to the $\beta$-phase as determined by the pcT method.

\section{Requirements for a combined measurement}

Precise combined pcT and DSC measurements require a small system volume with respect to the amount of gas desorbed but the sample amount should be big enough to have a minimum of hydrogen to be measured in the pcT system. In the DSC not the whole measuring system should be under pressure because of the high thermal conductivity of hydrogen $\left[\lambda_{\mathrm{H} 2}=0.1856 \mathrm{~W} /(\mathrm{m} \mathrm{K})\right.$ versus $\lambda_{\mathrm{N} 2}=0.0256 \mathrm{~W} /(\mathrm{m} \mathrm{K})$ at $300 \mathrm{~K}$ (Ref. 12)], which influences the calibration factor of the instrument. Additionally, if the whole system is under pressure the system volume is also too big. In commercially available high-pressure DSCs, often the whole system is under pressure with the above mentioned disadvantage or they have pressurized sample and reference holders but the volume of the cells are either too small $(50 \mu \mathrm{l})$ or too big $(10$ $\mathrm{ml})$. For the small holders, not enough hydrogen can be desorbed from the sample to be measured with the pcT system and in the large holders the system volume would be too big.

\section{INSTRUMENTAL}

\section{A. Quantitative description}

The DSC presented here has been designed and constructed as a heat-flow instrument. In such a DSC the temperature of the sample and reference are measured outside the sample holders contrary to a differential thermal analysis (DTA), where the temperatures are measured within the material. Due to this difference, the signal of a DSC is a quantitative value and only a function of the instrument and temperature, whereas the signal of a DTA is semiquantitative because the thermal properties of the sample are superimposed. A description of the different types of instruments and the corresponding theory are given by Mraw. ${ }^{13}$ The signal $\Delta T$ from the instrument is the difference between the measured sample and reference temperature $\left(T_{\mathrm{sm}}, T_{\mathrm{rm}}\right)$ and is proportional to the heat flow difference $d q / d t=d q_{s} / d t$ $-d q_{r} / d t$ of the heat flow to the sample and reference

$$
\Delta T=T_{\mathrm{rm}}-T_{\mathrm{sm}}=R_{\mathrm{th}}\left(\frac{d q_{s}}{d t}-\frac{d q_{r}}{d t}\right)=R_{\mathrm{th}} \frac{d q}{d t}=\frac{1}{f} P,
$$

where $R_{\mathrm{th}}$ is the thermal resistance of the heat flow path and its inverse value can be defined as the calibration factor $f$. For simplicity and because the heat flow difference $d q / d t$ has the dimensions of a power, it is defined as power $P$. According to the equation above there is the simple relation $P=f \cdot \Delta T$.

The temperature difference $\Delta T$ is dependent on the thermal properties of the sample and reference and is proportional to the difference in their heat capacities $C_{s}$ and $C_{r}$. Furthermore it is proportional to the heating rate $d T / d t$

$$
\Delta T=R_{\mathrm{th}} \frac{d T}{d t}\left(C_{s}-C_{r}\right) .
$$

The difference in power $P$ is the quantity to be measured; therefore $f$ has to be determined by an instrument calibration. For a real instrument $f$ is a function of the furnace temperature $T_{F}$ and the power $P_{s}$ released in the sample and consequently $f=f\left(T_{F}, P_{s}\right)$. 


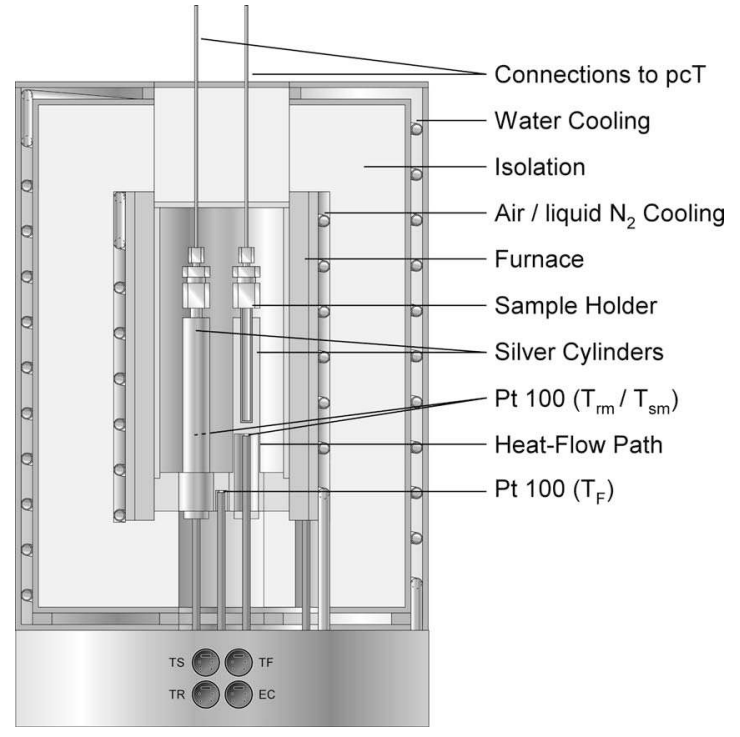

FIG. 1. Layout of the high-pressure and high-temperature DSC. Measured reference and sample temperature $\left(T_{\mathrm{rm}}, T_{\mathrm{sm}}\right)$, furnace temperature $\left(T_{F}\right)$.

\section{B. Setup}

In the DSC described here, the calorimetric block, heat flow path and sample/reference cylinders are made of the silver/copper alloy $\mathrm{Ag} 935$ consisting of $93.5 \% \mathrm{Ag}$ and of $6.5 \% \mathrm{Cu}$, with a melting range of $830-920{ }^{\circ} \mathrm{C}$. In order to simplify the design, the thermal properties such as the heat capacity and the thermal resistances of the calorimetric block parts are customized by their geometrical dimensions and not with different materials. The overall design of the DSC is represented in Fig. 1.

The sample and reference cylinders in which the stainless steel sample holders are placed are cylinders with two bores, one at the top where the sample and reference holder are introduced and one at the bottom where the temperature sensors are fixed (Fig. 1). The sample/reference holders are almost fully surrounded by the sample/reference cylinders in order to have a good thermal contact. The central part is the thin walled heat flow path with a well defined thermal resistance $R$ [cf. Eq. (3)], which mainly defines the properties of the instrument and makes it quantitative as described above. ${ }^{13}$ The sample/reference cylinders are connected with the silver block at the bottom and surrounded with a silver wall which is brazed with Silver Flo 20 (Ref. 14) that has a melting range from $776-815{ }^{\circ} \mathrm{C}$. The calorimetric block is completed with a silver lid.

The cylindrical electric heater around the calorimetric block with a power of $700 \mathrm{~W}$ is surrounded by cooling tubes for air or liquid nitrogen cooling. The furnace is isolated and at the outer part of the instrument is cooled with water cooling tubes.

The temperature of the calorimetric block is measured at the bottom with a Pt 100 sensor (tolerance 1/3 class B) which ceramic side is active brazed ${ }^{15}$ in an argon atmosphere with an active brazing alloy (Incusil-ABA, ${ }^{16}$ melting range of $605-715{ }^{\circ} \mathrm{C}$ ) onto the silver alloy surface. The temperature of the sample and reference are measured with the same type of Pt 100 sensors and are active brazed in the bottom bore of the sample/reference cylinders. The upper temperature limit of the DSC is $600{ }^{\circ} \mathrm{C}$ defined by the melting range of the Incusil-ABA $\left(605-715{ }^{\circ} \mathrm{C}\right)$. All the temperature sensors are wired in the four-wire arrangement and connected to a PT104 data Logger from Pico Technology with a resolution of $0.001{ }^{\circ} \mathrm{C}$ and an accuracy of $0.005^{\circ} \mathrm{C}$.

The sample and reference holder consists of bottom welded Swagelok stainless steel tubes with an outer diameter of $6 \mathrm{~mm}$ and different wall thicknesses depending on the pressure range $(0.5 \mathrm{~mm}$ for $170 \mathrm{bar}$ and $1 \mathrm{~mm}$ for $420 \mathrm{bar}$ at room temperature). For higher temperatures the maximal pressures have to be multiplied with a temperature factor, e.g., by 0.76 at $537{ }^{\circ} \mathrm{C}$ for AISI 316 stainless steel. ${ }^{17}$ The usable volume of the thin sample holder is $1 \mathrm{~cm}^{3}$. The sample and reference holders are connected to the pcT System with Swagelok reducing fittings to thinner tubes $(1 / 16$ inch) in order to have a high thermal resistance. The data acquisition and the whole system (DSC and pcT) are controlled with LABVIEW.

\section{INSTRUMENT PERFORMANCE}

\section{A. Calibration}

In a first step the instrument was calibrated for the temperature, the dependence of the calibration factor $f\left(P_{s}, T_{F}\right)$ [Eq. (3)] on the power released in the sample $P_{s}$ (dynamic range) and the furnace temperature $T_{F}$.

\section{Temperature calibration}

The temperature calibration was made with an indium (6 g, 99.999\% purity) and a zinc (3 g, 99.999\% purity) sample by measuring the onset temperature of the melting peaks and making a linear correction (not shown here).

\section{Dynamic range}

In order to determine the calibration factor $f\left(P_{s}, T_{F}\right)$ an exothermic sample event was simulated with an electric heater placed in the sample holder. Different electric square power peaks $P_{s}$ between $10 \mathrm{~mW}$ and $10 \mathrm{~W}$ were applied for 10 min at constant furnace temperature $T_{F}$ and the electric energy was determined by integrating the electric power with a power analyzer.

The signal $\Delta T$ as a function of the different applied electric powers $P_{s}$ at $T_{F}=100{ }^{\circ} \mathrm{C}$ is shown on a logarithmic scale

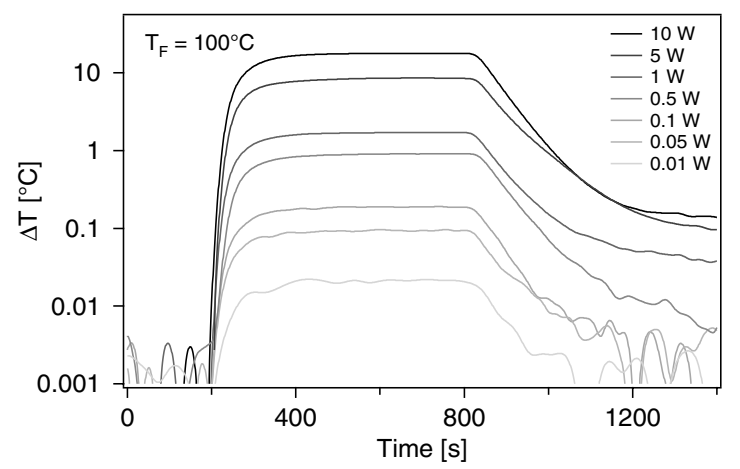

FIG. 2. DSC signal $\Delta T$ at $T_{F}=100{ }^{\circ} \mathrm{C}$ as a function of time for different applied square power peaks $P_{s}$ ranging from 0.01 to $10 \mathrm{~W}$. 


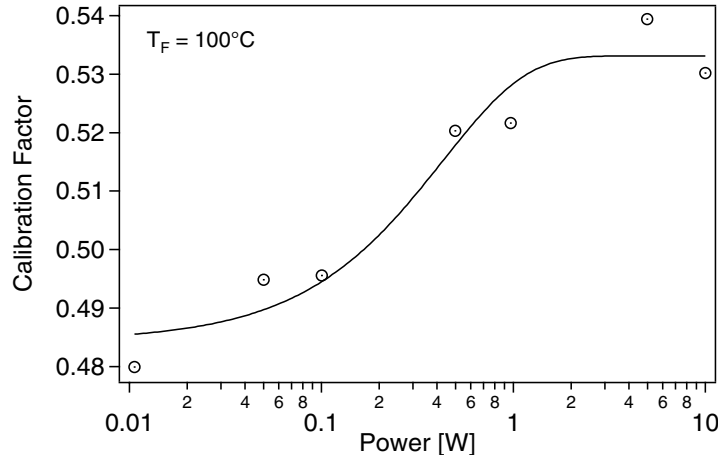

FIG. 3. Calibration Factor $f\left(P_{s}\right)$ (ratio of the applied energy and the measured time integrated DSC signal $\Delta T$ ) as a function of the applied power of the square peaks $P_{s}$ measured at $T_{F}=100{ }^{\circ} \mathrm{C}$. The fitted line is of the form $f\left(P_{s}\right)=f\left(10 \mathrm{~W}, 100{ }^{\circ} \mathrm{C}\right)+a_{1} \cdot \exp \left(a_{2} \cdot P_{s}\right)$.

in Fig. 2. By integration of Eq. (3), the calibration factor $f$ can be described as the ratio between the applied electric energy and the time integrated signal $\Delta T$. In Fig. 3 the calibration factor $f$ determined from the peaks in Fig. 2 is plotted as a function of the applied electric power $P_{s}$. For $P_{s}$ $>1 \mathrm{~W}$ the calibration factor is constant and is decreasing for $P_{s}<1 \mathrm{~W}$. An exponential function of the form $f\left(P_{s}\right)$ $=f\left(10 \mathrm{~W}, 100{ }^{\circ} \mathrm{C}\right)+a_{1} \cdot \exp \left(a_{2} \cdot P_{s}\right)$ was used to fit the data. The calibration factor as a function of the furnace temperature is shown in Fig. 4 for three different powers $P_{s}$. The calibration factor $f$ increases as a function of the furnace temperature $T_{F}$. The points can be fitted with a polynomial function of the form $f\left(T_{F}\right)=f\left(P_{s}\right)+a_{3} \cdot T_{F}^{3}$. As $a_{3}$ varies little as a function of $P_{s}$, it was held constant. The total calibration factor $f\left(P_{s}, T_{F}\right)$ as a function of the temperature and the power can finally be described by

$$
f\left(P_{s}, T_{F}\right)=f\left(10 \mathrm{~W}, 100{ }^{\circ} \mathrm{C}\right)+a_{1} \cdot \exp \left(a_{2} \cdot P_{s}\right)+a_{3} \cdot T_{F}^{3} .
$$

\section{Heating rates}

The DSC signal as a function of different heating rates $\left(0.5-5{ }^{\circ} \mathrm{C} / \mathrm{min}\right)$ for $6 \mathrm{~g}$ indium at $1 \mathrm{bar}$ of hydrogen is shown in Fig. 5. The average value is $28.40 \pm 0.11 \mathrm{~J} / \mathrm{g}$ corresponding to a small relative error of $0.4 \%$.

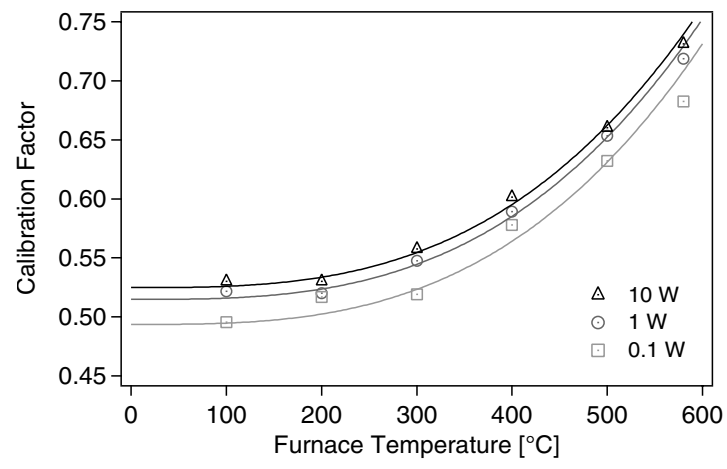

FIG. 4. Calibration Factor $f\left(T_{F}\right)$ (ratio of the applied energy and the measured time integrated DSC signal $\Delta T$ ) as a function of the temperature $T_{F}$ for three different square power peaks $P_{s}(0.1,1$, and $10 \mathrm{~W})$. The fitted lines are of the form $f\left(T_{F}\right)=f\left(P_{s}\right)+a_{3} \cdot T_{F}^{3}$ whereas the parameter $a_{3}$ was fixed to be the same for all tree curves.

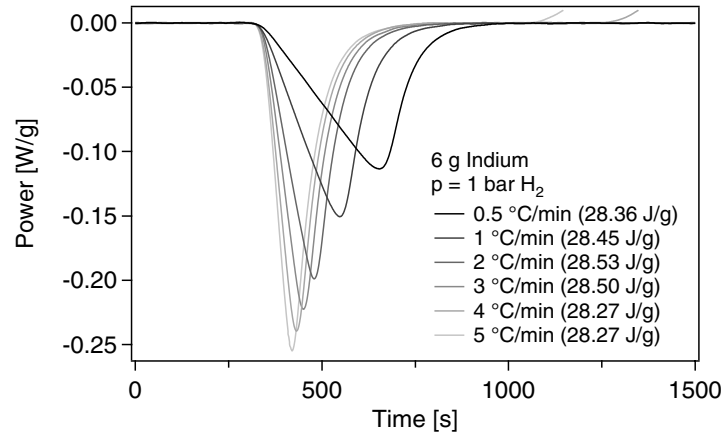

FIG. 5. Measurements of the melting of $6 \mathrm{~g}$ indium at one bar hydrogen as a function of time for different heating rates (from 0.5 to $5{ }^{\circ} \mathrm{C} / \mathrm{min}$ ). The melting enthalpies are relative to the value at $1{ }^{\circ} \mathrm{C} / \mathrm{min}$. For clarity the curves are shifted in order that the phase transformation occurs at the same time.

\section{Time constant}

The time constant of the instrument which is the time until the signal decreases by a factor of $1 / e$ is $90 \mathrm{~s}$, measured at the power peaks shown in Fig. 2.

\section{Pressure independence}

The hydrogen pressure dependence is validated by melting peaks of indium as a function of the applied hydrogen pressure (vacuum to 100 bar) and is shown in Fig. 6. Clearly the integrated power of the indium sample is independent of the hydrogen pressure (average value of $28.41 \pm 0.06 \mathrm{~J} / \mathrm{g}$ ). This hydrogen pressure independence of the calibration factor is the big advantage of this instrument design and allows making measurements under varying hydrogen pressures.

\section{Noise}

The noise of the DSC was determined by the standard deviation of the signal measured at a constant temperature of $60{ }^{\circ} \mathrm{C}$ and is $1 \mathrm{~mW}$.

\section{B. Measurements of $\mathrm{LaNi}_{5}$}

The main novelty is the combination of pcT and DSC in the same measurement, therefore in a second step the functionality of the instrument was shown with a combined pcT and DSC measurements using $\mathrm{LaNi}_{5}$ as a benchmark mate-

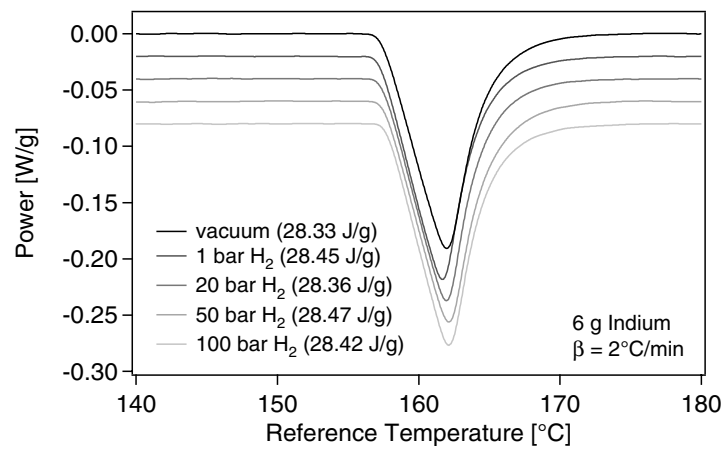

FIG. 6. Measurements of the melting of $6 \mathrm{~g}$ indium at a heating rate of $2{ }^{\circ} \mathrm{C} / \mathrm{min}$ as a function of the reference temperature for different hydrogen pressures (vacuum to $100 \mathrm{bar}$ ). The melting enthalpies are relative to the value at 1 bar hydrogen. For clarity the curves are shifted vertically. 


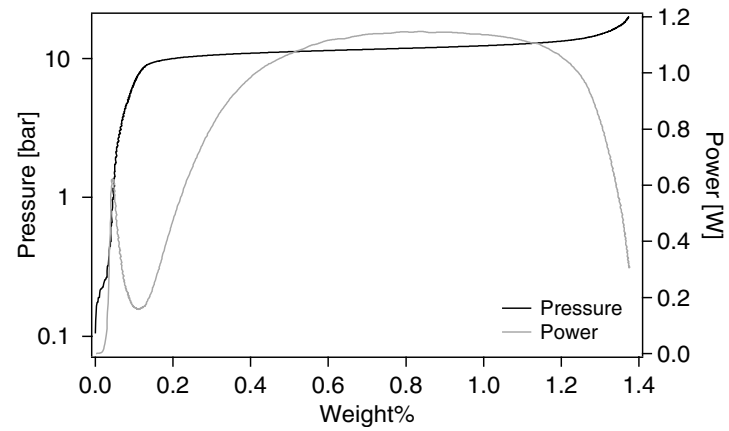

FIG. 7. Combined pcT and DSC measurement of the hydrogenation of $\mathrm{LaNi}_{5}$ measured at a flow of $5 \mathrm{SCCM}$. The pressure and the power are plotted as a function of the hydrogen concentration.

rial. Sample preparation consisted of two hydrogenation and dehydrogenation cycles at $60{ }^{\circ} \mathrm{C}$ and 100 bar hydrogen in order to activate the $\mathrm{LaNi}_{5}(4.142 \mathrm{~g})$.

For the measurement at $60{ }^{\circ} \mathrm{C}$, five different flow rates from 10 to 50 SCCM (SCCM denotes standard cubic centimeters per minute at STP) were applied. In Fig. 7 a pcT isotherm of the hydrogen absorption of $\mathrm{LaNi}_{5}$ measured at $60{ }^{\circ} \mathrm{C}$ and at a flow of $50 \mathrm{SCCM}$ is shown with the corresponding DSC signal. The first peak at a low hydrogen concentration is presumably due to some kind of surface phenomenon as, e.g., chemisorption suggested by Murray et al. ${ }^{11}$ The second, broad peak corresponds to the growth of the $\beta$-phase out of the $\alpha$-phase in the two phase region. ${ }^{10}$ The power measurements for the ab- and desorptions for the different flows are shown in Fig. 8 as a function of time. The power was recorded until the reaction was equilibrated and reached a value of zero. The integrated and normalized powers are plotted in Fig. 9, whereas the horizontal lines correspond to the average values. As a function of the flow rate the absorption enthalpy is almost constant and the average value of $-31.3 \pm 0.2 \mathrm{~kJ} / \mathrm{mol} \mathrm{H}_{2}$ (statistical error) corresponds well to the integrated enthalpy of hydriding of $-32.1 \pm 0.1 \mathrm{~kJ} / \mathrm{mol} \mathrm{H}_{2}$ measured by Hubbard et al. ${ }^{10}$ at $25^{\circ} \mathrm{C}$. The enthalpy of desorption is also constant as a function of the flow rate with slight fluctuations and the average value is $30.5 \pm 0.3 \mathrm{~kJ} / \mathrm{mol} \mathrm{H}_{2}$. Hubbard et al. ${ }^{10}$ do not give an integrated value of the enthalpy of desorption but only a value on the plateau corresponding to the transformation of the $\beta$ - to $\alpha$-phase of $31.4 \mathrm{~kJ} / \mathrm{mol} \mathrm{H}_{2}$ is given. The logarithms of the plateau pressures divided by $p_{0}$ at different flow rates

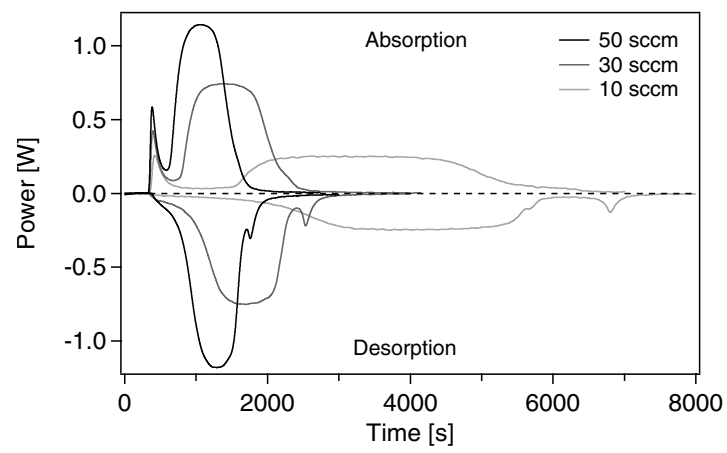

FIG. 8. DSC power as a function of time for the ab- and desorption of $\mathrm{LaNi}_{5}$ for different hydrogen flows (10-50 SCCM).

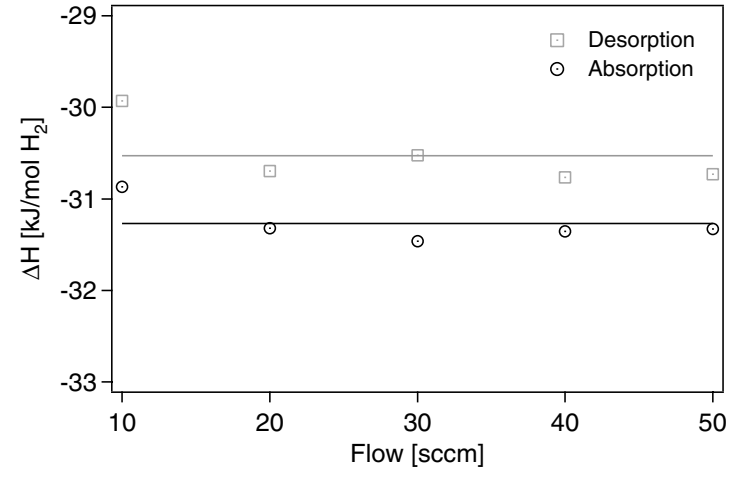

FIG. 9. Enthalpy of the ab- and desorption calculated from the integration and normalization of the curves shown in Fig. 8 plotted as a function of the hydrogen flow. The horizontal lines correspond to the average values.

for the ab- and desorption are shown in Fig. 10. The values are not constant as a function of the flow rate. If the values are fitted to a straight line and extrapolated to a flow of 0 SCCM the equilibrium pressures are obtained. The absorption equilibrium pressure is $p_{\mathrm{eq}}{ }^{\mathrm{abs}}=9.73 \pm 0.07$ bar and for the desorption $p_{\mathrm{eq}}^{\text {des }}=7.9 \pm 0.1$ bar. Taking the integrated values of $\Delta H$ determined above, this leads according to the Van 't Hoff equation to the following entropies: $\Delta S_{\mathrm{abs}}=$ -113 and $\Delta S_{\mathrm{des}}=109 \mathrm{~J} /\left(\mathrm{K} \mathrm{mol} \mathrm{H}_{2}\right)$.

\section{CONCLUSIONS}

The construction of a high-pressure (200 bar) and hightemperature $\left(600{ }^{\circ} \mathrm{C}\right) \mathrm{DSC}$ for the combined use with a pcT instrument was shown. The big advantage of the instrument is that only the sample and reference holder are pressurized and consequently the calibration factor is independent of the hydrogen pressure. With the combination of the DSC with a pcT system, simultaneous thermodynamic and volumetric measurements become accessible. Furthermore structural phase transformations and hydrogen sorption events can be separated and identified. The instrument was calibrated and successfully tested with the hydrogen ab- and desorption reaction of $\mathrm{LaNi}_{5}$. The determined thermodynamic parameters are in agreement with literature values.

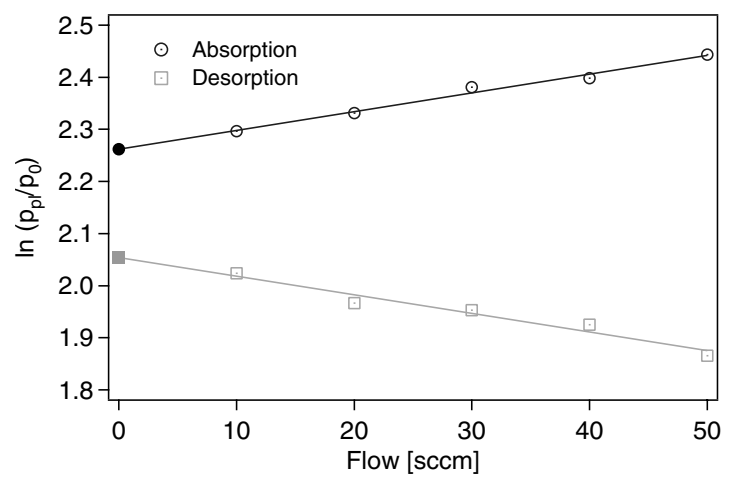

FIG. 10. $\ln \left(p_{\mathrm{pl}} / p_{0}\right)$ as a function of the hydrogen flow for the ab- and desorption of $\mathrm{LaNi}_{5}$ at $60{ }^{\circ} \mathrm{C}$. The values are linearly extrapolated to the equilibrium pressure $p_{\mathrm{eq}}$ at a flow of 0 SCCM (filled symbols). 


\section{ACKNOWLEDGMENTS}

This work was financially supported by the Helmholtz Initiative "Functional Materials for Mobile Hydrogen Storage FuncHy" and is greatly acknowledged. The work of the workshop at the University of Fribourg, the construction department and the mechanical workshop at Empa is also gratefully acknowledged.

${ }^{1}$ L. Schlapbach and A. Züttel, Nature (London) 414, 353 (2001).

${ }^{2}$ J. H. N. van Vucht, F. A. Kuijpers, and H. C. A. M. Bruning, Philips Res. Rep. 25, 133 (1970).

${ }^{3}$ http://hydpark.ca.sandia.gov/.

${ }^{4}$ M. Bielmann, S. Kato, Ph. Mauron, and A. Züttel, Rev. Sci. Instrum. 80, 083901 (2009).

${ }^{5}$ E. Flüglein and A. Léon, in Hydrogen Technology, Mobile and Portable Applications, edited by A. Léon (Springer, New York, 2008), p. 516.
${ }^{6}$ C. Rongeat, I. Llamas-Jansa, S. Doppiu, S. Deledda, A. Borgschulte, L. Schultz, and O. Gutfleisch, J. Phys. Chem. B 111, 13301 (2007).

${ }^{7}$ A. Borgschulte, U. Bösenberg, G. Barkhordarian, M. Dornheim, and R. Bormann, Catal. Today 120, 262 (2007).

${ }^{8}$ G. W. H. Höhne, W. F. Hemminger, and H.-J. Flammersheim, Differential Scanning Calorimetry, 2nd ed. (Springer, New York, 2003).

${ }^{9}$ H. Wenzl and E. Lebsanft, J. Phys. F: Met. Phys. 10, 2147 (1980).

${ }^{10}$ W. N. Hubbard, P. L. Rawlins, P. A. Connick, R. E. Stedwell, and P. A. G. O’Hare, J. Chem. Thermodyn. 15, 785 (1983).

${ }^{11}$ J. J. Murray, M. L. Post, and J. B. Taylor, J. Less-Common Met. 80, 211 (1981).

${ }^{12}$ www.nist.gov.

${ }^{13}$ S. C. Mraw, in CINDAS Data Series on Material Properties, edited by C. Y. Ho (Hemisphere, New York, 1988), Vols. 1-2, p. 395.

${ }^{14}$ www.jm-metaljoining.com.

${ }^{15}$ V. Bissig, M. Galli, and J. Janczak-Rusch, Adv. Eng. Mater. 8, 191 (2006).

${ }^{16}$ www.wesgometals.com.

${ }^{17}$ www.swagelok.com. 